\title{
Extra-Axial Tumors
}

Extra-axial tumors most commonly arise from the meninges, calvarium, or skull base. Localization of a lesion as extra-axial or extra-cerebral in origin, has significant clinical importance in terms of treatment planning and predicting prognosis. High-resolution fat-suppressed imaging is crucial for specific evaluation of the skull base, and correlation with computed tomography (CT) is often helpful in these cases. This unit presents the set of magnetic resonance sequences used for imaging extra-axial tumors found in specific locations; sequence modifications will be discussed where necessary. The sequences described in this unit are based on the authors' experience with a $1.5 \mathrm{~T}$ scanner (GE Medical Systems), but can be expected to be equally applicable to other field strengths and scanners from other manufacturers.

\section{RULE OUT (R/O) EXTRA-AXIAL TUMOR}

Spin-echo and fast spin-echo imaging (sequences 1 to 4 ) essentially provide all of the information needed to make an accurate diagnosis when evaluating for primary extra-axial tumors. The fluid-attenuated inversion recovery (FLAIR) images (sequence 4) and post-contrast $T_{1}$-weighted images (sequence 5) are most sensitive for evaluating tumor spread particularly along the subarachnoid space (leptomeninges; Singer et al., 1998). Sequences 1 to 5 comprise the preferred protocol.

Table A3.4.1 presents equipment parameters for imaging brain tumors.

NOTE: Be sure that technologists and nurses have immediate access to any equipment such as crash carts or oxygen that may be necessary in the event of an emergency. Contrast reactions are rare, but the resources are necessary.

\section{Materials}

Intravenous contrast agent (e.g., Magnevist, Omniscan, Prohance)

Normal saline $(0.9 \% \mathrm{NaCl})$, sterile

\section{Set up patient and equipment}

1. Interview (screen) the patient to ensure that he or she has no contraindications such as cardiac pacemaker or other implants containing ferromagnetic materials. Also be sure to find out if the patient has any health conditions that may require the presence of specific emergency equipment during the scanning procedure, or necessitate any other precautions.

Generally standard screening forms are used for all patients scanned in a magnetic resonance system.

The presence of any ferromagnetic metals may be a health hazard to the patient when he or she is inside the magnet, and will also affect the imaging. If in doubt as to the exact

Table A3.4.1 Equipment Parameters for Imaging Brain Tumors

\begin{tabular}{ll}
\hline Coil Type & Quadrature head coil \\
Gradient coil strength & $25 \mathrm{mT} / \mathrm{m}$ (or whatever the system permits) \\
Cardiac gating & No \\
Peripheral gating & For safety only \\
Respiratory gating & No \\
Respirator & If required by patient \\
Oxygen & If required by patient \\
Motion cushions & No
\end{tabular}

Contributed by Annette O. Nusbaum and Scott W. Atlas

Current Protocols in Magnetic Resonance Imaging (2001) A3.4.1-A3.4.11

Copyright @ 2001 by John Wiley \& Sons, Inc.
BASIC

PROTOCOL

Cerebral Neoplastic Disease

A3.4.1 
composition of the items, it is best to exclude patients with metal implants; see Shellock (1996) for discussion of what implants may be safely scanned using magnetic resonance.

Patients may be accompanied into the magnet room by a friend or family member, who can sit in the room during the scan and comfort the patient as needed. This companion must be screened as well to ensure the absence of loose metal objects on the body or clothing.

2. If the procedure is a research protocol, have the patient sign any necessary consent form.

3. Have the patient remove all jewelry and change into a gown to eliminate any metal that might be found in clothing.

4. Have the patient wash off any mascara and other makeup to avoid local tissue heating and image artifacts.

5. Inform the patient about what will occur during the procedure, what he or she will experience in the magnet, and how to behave, including the following:

a. If earphones or headphones are used to protect the ears from the loud sounds produced by the gradients, the patient will be asked to wear these, but will be able to communicate with you at any time during the imaging.

b. The patient will be given a safety squeeze-bulb or similar equipment to request assistance at any time (demonstrate how this works).

c. For good results the patient should not talk, and should avoid or minimize swallowing or other movement, during each scan-i.e., as long as the banging sounds continue. Between scans, talking and swallowing are allowed in most cases, but should be avoided when comparative positional studies are being performed; the patient will be informed when this is the case.

d. Nevertheless, the patient may call out at any time if he or she feels it necessary.

6. Have the patient lie in the supine position on the table. Setup all monitoring equipment before or right after the patient lies down.

7. Center patient in the head coil at the region where key information is desired. Constrain the head and neck to prevent motion. Generally, fix the patient's head so that the head is horizontal (not tilted) and the neck and head lie along the axis of the patient table.

8. If needed, place a pillow or other support under the knees to make the patient more comfortable.

9. Use the centering light to position the patient (centered on the nasion) and put him or her into the center of the magnet.

Once this step has been performed, so long as the patient does not move on the table, the table itself can be moved and then replaced in the same position as before without jeopardizing the positioning of one scan relative to another.

10. If the patient is unable to hold still, provide an appropriate sedative.

\section{Sequence 1: Localizer}

11. Run sequence 1 according to Table A3.4.2.

\section{Sequence 2: $T_{1}$-weighted scan}

12. Run sequence 2 according to Table A3.4.3. 
Table A3.4.2 Primary Clinical Imaging Parameters for Sequence 1 (Localizer; $T_{1}$-Weighted)

$\begin{array}{ll}\text { Patient position } & \text { Supine } \\ \text { Scan type } & \text { Spin echo } \\ \text { Imaging plane (orientation) } & \text { Sagittal } \\ \text { Central slice or volume center } & \text { Laser light centered on nasion } \\ \text { Echo time }\left(T_{\mathrm{E}}\right) & \begin{array}{l}11 \mathrm{msec} \text { (or select "minimum } \\ \text { full" echo time) }\end{array} \\ \text { Receiver bandwidth }(\mathrm{RBW}) & 10 \mathrm{kHz} \\ \text { Repeat time }\left(T_{\mathrm{R}}\right) & 500 \mathrm{msec} \\ \text { Flip angle }(\mathrm{FA}) & 90^{\circ} \\ \text { Fields of view }\left(\mathrm{FOV}, \mathrm{x}, \mathrm{FOV}_{\mathrm{y}}\right) & 240 \mathrm{~mm}, 240 \mathrm{~mm} \\ \text { Resolution }(\Delta x, \Delta y) & 0.94 \mathrm{~mm}, 1.25 \mathrm{~mm} \\ \text { Number of data points collected }\left(N_{\mathrm{x}}, N_{\mathrm{y}}\right) & 256,192 \\ \text { Slice thickness }(\Delta z) & 5 \mathrm{~mm} \\ \text { Number of slices } & 20(\text { or as many as needed to cover } \\ \text { the region of interest) } & 2 \mathrm{~mm} \\ \text { Slice gap } & 1 \\ \text { Number of acquisitions }\left(N_{\mathrm{acq}}\right) & \text { No } \\ \text { Swap read and phase encoding } & \text { Not applicable } \\ \text { Saturation pulses } & \sim 1 \mathrm{~min}, 36 \text { sec } \\ \text { Scan time } & \end{array}$

Table A3.4.3 Primary Clinical Imaging Parameters for Sequence 2 ( $T_{1}$-Weighted)

\begin{tabular}{ll}
\hline Patient position & Supine \\
Scan type & Spin echo \\
Imaging plane (orientation) & Transverse \\
Central slice or volume center & Laser light centered on nasion \\
Echo time $\left(T_{\mathrm{E}}\right)$ & $11 \mathrm{msec}$ (or select "minimum \\
& full" echo time) \\
Receiver bandwidth $(\mathrm{RBW})$ & $10 \mathrm{kHz}$ \\
Repeat time $\left(T_{\mathrm{R}}\right)$ & $500 \mathrm{msec}$ \\
Flip angle $(\mathrm{FA})$ & $90^{\circ}$ \\
Fields of view $\left(\mathrm{FOV}_{\mathrm{x}}, \mathrm{FOV}_{\mathrm{y}}\right)$ & $240 \mathrm{~mm}, 240 \mathrm{~mm}$ \\
Resolution $(\Delta x, \Delta y)$ & $0.94 \mathrm{~mm}, 1.25 \mathrm{~mm}$ \\
Number of data points collected $\left(N_{\mathrm{x}}, N_{\mathrm{y}}\right)$ & 256,192 \\
Slice thickness $(\Delta z)$ & $5 \mathrm{~mm}$ \\
Number of slices & 20 (or as many as needed to cover \\
& the region of interest) \\
Slice gap & $2.5 \mathrm{~mm}$ \\
Number of acquisitions $\left(N_{\mathrm{acq}}\right)$ & 1 \\
Swap read and phase encoding & Yes \\
Saturation pulses & Not applicable \\
Scan time & $\sim 1 \mathrm{~min}, 36$ sec \\
\hline
\end{tabular}


Sequence 3: $T_{2}$-weighted scan

13. Run sequence 3 according to Table A3.4.4.

\section{Sequence 4: Fluid-attenuated inversion recovery (FLAIR) scan}

14. Run sequence 4 according to Table A3.4.5.

\section{Sequence 5: Post-contrast imaging}

15. Remove the patient from the scanner. Do not allow the patient to move on the table. Establish an intravenous line from for injection of contrast agent. Attach this line securely to the patient so that movement into or out of the magnet will not pull at the patient's arm. Move the patient back into the scanner.

It is preferable to insert the line prior to imaging and to leave the patient in the magnet with no intervening motion between the scans run before contrast agent injection and those run after injection.

16. Leaving the patient in the magnet, inject the contrast agent, flush the line with $10 \mathrm{ml}$ saline.

A dose of $0.1 \mathrm{mmol} / \mathrm{kg}$ of contrast agent is usually given.

A delay in scanning may actually be beneficial when evaluating for metastases, which is one of the reasons we scan in multiple planes after intravenous contrast administration.

17. Acquire the post-contrast images using the same parameters as in sequence 2 ( $T_{1}$-weighted scan; Table A3.4.3).

In addition to the transverse plane, the coronal plane is routinely obtained, with the following changes to the parameters in sequence $2:$ (a) flow compensation is on; (b) $T_{E}$ is 20 msec (prolonged due to flow compensation gradients); and (c) it is not necessary to swap read and phase encoding directions. In patients requiring sedation and in all pediatric patients, we also routinely obtain a third post-contrast plane of imaging (sagittal).

Table A3.4.4 Primary Clinical Imaging Parameters for Sequence 3

( $T_{2}$-Weighted)

Patient position

Scan type

Imaging plane (orientation)

Central slice or volume center

Echo time $\left(T_{\mathrm{E}}\right)$

Receiver Bandwidth (RBW)

Echo train length (ETL)

Repeat time $\left(T_{\mathrm{R}}\right)$

Flip angle (FA)

Fields of view $\left(\mathrm{FOV}_{\mathrm{x}}, \mathrm{FOV}_{\mathrm{y}}\right)$

Resolution $(\Delta x, \Delta y)$

Number of data points collected $\left(N_{\mathrm{x}}, N_{\mathrm{y}}\right)$

Slice thickness $(\Delta z)$

Number of slices

Slice gap

Number of acquisitions $\left(N_{\text {acq }}\right)$

Swap read and phase encoding

Saturation pulses

Scan time
Supine

Fast spin echo

Transverse

Laser light centered on nasion

$102 \mathrm{msec}$ (effective)

$16 \mathrm{kHz}$

8

$3600 \mathrm{msec}$

$90^{\circ}$

$240 \mathrm{~mm}, 240 \mathrm{~mm}$

$0.94 \mathrm{~mm}, 1.25 \mathrm{~mm}$

256, 192

$5 \mathrm{~mm}$

20 (or as many as needed to cover the region of interest)

$2.5 \mathrm{~mm}$

1

Yes

Not applicable

$\sim 1 \mathrm{~min}, 30 \mathrm{sec}$ 
Table A3.4.5 Primary Clinical Imaging Parameters for Sequence 4 (FLAIR)

\begin{tabular}{|c|c|}
\hline Patient position & Supine \\
\hline Scan type & Inversion-recovery fast-spin echo \\
\hline Imaging plane (orientation) & Transverse \\
\hline Central slice or volume center & Laser light centered on nasion \\
\hline Echo time $\left(T_{\mathrm{E}}\right)$ & $120 \mathrm{msec}$ (effective) \\
\hline Receiver Bandwidth (RBW) & $16 \mathrm{kHz}$ \\
\hline Echo train length (ETL) & 8 \\
\hline Repeat time $\left(T_{\mathrm{R}}\right)$ & $10,000 \mathrm{msec}$ \\
\hline Inversion time $\left(T_{\mathrm{I}}\right)$ & $2200 \mathrm{msec}$ \\
\hline Flip angle (FA) & $180^{\circ}$ \\
\hline Fields of view $\left(\mathrm{FOV}_{\mathrm{x}}, \mathrm{FOV}_{\mathrm{y}}\right)$ & $240 \mathrm{~mm}, 240 \mathrm{~mm}$ \\
\hline Resolution $(\Delta x, \Delta y)$ & $0.94 \mathrm{~mm}, 1.25 \mathrm{~mm}$ \\
\hline Number of data points collected $\left(N_{\mathrm{x}}, N_{\mathrm{y}}\right)$ & 256,192 \\
\hline Slice thickness $(\Delta z)$ & $5 \mathrm{~mm}$ \\
\hline Number of slices & $\begin{array}{l}20 \text { (or as many as needed to cover } \\
\text { the region of interest) }\end{array}$ \\
\hline Slice gap & $2.5 \mathrm{~mm}$ \\
\hline Number of acquisitions $\left(N_{\mathrm{acq}}\right)$ & 1 \\
\hline Swap read and phase encoding & Yes \\
\hline Saturation pulses & Not applicable \\
\hline Scan time & $\sim 5 \min$ \\
\hline
\end{tabular}

\section{MENINGIOMA}

PROTOCOL 1

The majority of meningiomas are found incidentally and are located in the supratentorial compartment (along the falx or cerebral convexity). Sequences 1 to 5 (see Basic Protocol) comprise the preferred protocol. The location of the lesion determines whether further sequences should be obtained (i.e., if the tumor is adjacent to the orbit or internal auditory canals, then additional sequences examining the specific region should be obtained). Not uncommonly, meningiomas may be inseparable from the adjacent dural venous sinus and the question arises whether or not the tumor has invaded the venous sinus. In these cases, magnetic resonance venography (MRV) is useful to confirm whether or not the venous sinus is patent (see Chapter A2 for evaluating the cerebral venous sinuses).

For procedure, see Basic Protocol.

\section{NERVE SHEATH TUMORS-E.G., ACOUSTIC NEUROMA}

Patients with nerve sheath tumors typically present with sensorineural hearing loss, arise within the vestibular or acoustic portions of cranial nerve VIII, and are found within the internal auditory canals. High-resolution noncontrast (sequence 6) and post-contrast imaging with fat suppression (sequence 7) is essential to evaluate this region. Imaging of the entire brain with pre-contrast (sequences 1,3, and 4) and post-contrast sequences (sequence 5; see Basic Protocol) is a standard component of the complete evaluation of these patients.

\section{Set up patient and equipment}

1. Use the same equipment and perform the same patient setup as for the previous method (see Basic Protocol, steps 1 to 10).

2. Run sequences 1, 3, and 4 in Basic Protocol.

Cerebral

Neoplastic Disease

A3.4.5 
Table A3.4.6 Primary Clinical Imaging Parameters for Sequence 6 ( $T_{2}$-Weighted)

Patient position

Scan type

Imaging plane (orientation)

Central slice or volume center

Echo time $\left(T_{\mathrm{E}}\right)$

Receiver Bandwidth (RBW)

Echo train length (ETL)

Repeat time $\left(T_{\mathrm{R}}\right)$

Flip angle (FA)

Fields of view $\left(\mathrm{FOV}_{\mathrm{x}}, \mathrm{FOV}_{\mathrm{y}}\right)$

Resolution $(\Delta x, \Delta y)$

Number of data points collected $\left(N_{\mathrm{x}}, N_{\mathrm{y}}\right)$

Slice thickness $(\Delta z)$

Number of slices

Slice gap

Number of acquisitions $\left(N_{\text {acq }}\right)$

Swap read and phase encoding

Saturation pulses

Fat suppression

Scan time
Supine

Fast spin echo

Transverse

Laser light centered on nasion

$102 \mathrm{msec}$ (effective)

$16 \mathrm{kHz}$

16

$4000 \mathrm{msec}$

$90^{\circ}$

$180 \mathrm{~mm}, 180 \mathrm{~mm}$

$0.70 \mathrm{~mm}, 0.70 \mathrm{~mm}$

256,256

$3 \mathrm{~mm}$

As many as needed to cover entire skull base

$0.5 \mathrm{~mm}$

3

Yes

None except fat

Yes

$3 \mathrm{~min}, 20 \mathrm{sec}$

Table A3.4.7 Primary Clinical Imaging Parameters for Sequence 7 (Post-Contrast $T_{1}$-Weighted)

Patient position

Scan type

Imaging plane (orientation)

Central slice or volume center

Echo time $\left(T_{\mathrm{E}}\right)$

Receiver Bandwidth (RBW)

Repeat time $\left(T_{\mathrm{R}}\right)$

Flip angle (FA)

Fields of view $\left(\mathrm{FOV}_{\mathrm{x}}, \mathrm{FOV}_{\mathrm{y}}\right)$

Resolution $(\Delta x, \Delta y)$

Number of data points collected $\left(N_{\mathrm{x}}, N_{\mathrm{y}}\right)$

Slice thickness $(\Delta z)$

Number of slices

Slice gap

Number of acquisitions $\left(N_{\text {acq }}\right)$

Swap read and phase encoding

Saturation pulses

Fat suppression

Scan time

\section{Supine}

Spin echo

Transverse and coronal

Laser light centered on nasion

$20 \mathrm{msec}$

$10 \mathrm{kHz}$

$500 \mathrm{msec}$

$90^{\circ}$

$180 \mathrm{~mm}, 180 \mathrm{~mm}$

$0.70 \mathrm{~mm}, 0.94 \mathrm{~mm}$

256, 192

$3 \mathrm{~mm}$

As many as needed to cover the area of interest

$0.5 \mathrm{~mm}$

3

Yes, when it is transverse

None except fat

Yes

$5 \mathrm{~min}, 20 \mathrm{sec}$ 
Sequence 6: High resolution $T_{2}$-weighted scan

3. Run sequence 6 (high-resolution transverse $T_{2}$-weighted images through the internal auditory canals) according to Table A3.4.6. This sequence nicely depicts the VII and VIII cranial nerve roots.

\section{Sequence 7: High resolution $T_{1}$-weighted scan (post-contrast)}

4. Repeat steps 15 and 16 in the Basic Protocol.

5. Run sequence 7 according to Table A3.4.7. Following the administration of contrast, obtain sequence 7 in both the transverse and coronal planes through the internal auditory canals.

6. In addition, obtain transverse $T_{1}$-weighted images (sequence 5, step 17 in the Basic Protocol) through the entire brain.

\section{PITUITARY/SELLA TURCICA MASSES}

Pituitary/sella turcica lesions are best evaluated by dedicated high resolution imaging of the sella in the sagittal and coronal planes. Non-contrast imaging including high resolution sagittal $T_{1}$-weighted localizer (sequence 8), coronal $T_{1}$-weighted (sequence 9 ) and coronal $T_{2}$-weighted images (sequence 6 , with orientation changed to coronal and with no swap read and phase encoding directions) beautifully depict complicated anatomic regions. Contrast enhanced images (sequence 9) are essential and may demonstrate small pituitary lesions (microadenomas) not seen otherwise. Post-contrast imaging (sequence 5) of the entire brain is always performed.

\section{Set up patient and equipment}

1. Use the same equipment and perform the same patient setup as for the previous method (see Basic Protocol, steps 1 to 10).

\section{Sequence 8: $T_{1}$-weighted localizer}

2. Run sequence 8 (high resolution sagittal $T_{1}$-weighted localizer of the sella) according to Table A3.4.8.

Table A3.4.8 Primary Clinical Imaging Parameters for Sequence 8 ( $T_{1}$-Weighted Localizer)

\begin{tabular}{ll}
\hline Patient position & Supine \\
Scan type & Spin echo \\
Imaging plane (orientation) & Sagittal \\
Central slice or volume center & Laser light centered on nasion \\
Echo time $\left(T_{\mathrm{E}}\right)$ & $20 \mathrm{msec}$ \\
Receiver Bandwidth $(\mathrm{RBW})$ & $10 \mathrm{kHz}$ \\
Repeat time $\left(T_{\mathrm{R}}\right)$ & $500 \mathrm{msec}$ \\
Flip angle $(\mathrm{FA})$ & $90^{\circ}$ \\
Fields of view $\left(\mathrm{FOV}, \mathrm{F}_{\mathrm{x}}\right.$, FOV $\left.\mathrm{y}\right)$ & $160 \mathrm{~mm}, 160 \mathrm{~mm}$ \\
Resolution $(\Delta x, \Delta y)$ & $0.63 \mathrm{~mm}, 0.83 \mathrm{~mm}$ \\
Number of data points collected $\left(N_{\mathrm{x}}, N_{\mathrm{y}}\right)$ & 256,192 \\
Slice thickness $(\Delta z)$ & $3 \mathrm{~mm}$ \\
Number of slices & 20 \\
Slice gap & 0 \\
Number of acquisitions $\left(N_{\mathrm{acq}}\right)$ & 3 \\
Swap read and phase encoding & No \\
Saturation pulses & Not applicable \\
Slice series & Interleaved \\
Scan time & 5 min \\
\hline
\end{tabular}


ALTERNATE PROTOCOL 4

Extra-Axial

Tumors

Table A3.4.9 Primary Clinical Imaging Parameters for Sequence 9

( $T_{1}$-Weighted)

\begin{tabular}{ll}
\hline Patient position & Supine \\
Scan type & Spin echo \\
Imaging plane (orientation) & Coronal \\
Central slice or volume center & Laser light centered on nasion \\
Echo time $\left(T_{\mathrm{E}}\right)$ & $20 \mathrm{msec}$ \\
Receiver Bandwidth $(\mathrm{RBW})$ & $10 \mathrm{kHz}$ \\
Repeat time $\left(T_{\mathrm{R}}\right)$ & $500 \mathrm{msec}$ \\
Flip angle $(\mathrm{FA})$ & $90^{\circ}$ \\
Fields of view $\left(\mathrm{FOV}, \mathrm{FOV}_{\mathrm{y}}\right)$ & $180 \mathrm{~mm}, 180 \mathrm{~mm}$ \\
Resolution $(\Delta x, \Delta y)$ & $0.70 \mathrm{~mm}, 0.94 \mathrm{~mm}$ \\
Number of data points collected $\left(N_{\mathrm{x}}, N_{\mathrm{y}}\right)$ & 256,192 \\
Slice thickness $(\Delta z)$ & $3 \mathrm{~mm}$ \\
Number of slices & As many as needed to cover the \\
Slice gap & area of interest \\
Number of acquisitions $\left(N_{\mathrm{acq}}\right)$ & $0.5 \mathrm{~mm}$ \\
Swap read and phase encoding & 3 \\
Saturation pulses & No \\
Fat suppression & No \\
Scan time & No \\
\end{tabular}

3. Run sequence 3 (transverse $T_{2}$-weighted of the entire brain) in Basic Protocol.

\section{Sequence 9: High resolution $T_{1}$-weighted scan}

4. Run sequence 9 (coronal images through the sella) according to Table A3.4.9.

\section{Sequence 10: High resolution $T_{2}$-weighted scan}

5. Run sequence 6 (coronal $\mathrm{T}_{2}$-weighted images of the sella) based on Table A3.4.6.

6. Repeat steps 15 and 16 in the Basic Protocol.

7. Run sequence 9 (post-contrast coronal T1-weighted images through the sella) according to Table A3.4.9.

8. Run sequence 5 (transverse images of the entire brain).

\section{ARACHNOID CYST}

Arachnoid cysts are benign, developmental anomalies of the subarachnoid space and typically demonstrate signal characteristics identical to that of cerebrospinal fluid (CSF).

Occasionally, difficulties arise in differentiating an arachnoid cyst from an epidermoid cyst. FLAIR images or diffusion-weighted images may be useful to identify slight differences in signal intensity from that of CSF, which would indicate an epidermoid cyst, rather than an arachnoid cyst.

\section{Set up patient and equipment}

1. Follow steps 1 to 10 in the Basic Protocol and run sequences 1 to 4 .

\section{Sequence 11: Diffusion-weighted imaging (DWI)}

Multislice single-shot spin echo echo-planar diffusion-weighted sequences are obtained with two diffusion gradient pulses applied before and after the $180^{\circ}$ pulse. The pulse 
Table A3.4.10 Primary Clinical Imaging Parameters for Sequence 11 (DWI)

\begin{tabular}{ll}
\hline Patient position & Supine \\
Scan type & Diffusion-weighted EPI-spin echo \\
Imaging plane (orientation) & Transverse \\
Central slice or volume center & Laser light centered on nasion \\
Echo time $\left(T_{\mathrm{E}}\right)$ & $125 \mathrm{msec}$ \\
Repeat time $\left(T_{\mathrm{R}}\right)$ & $10,000 \mathrm{msec}$ \\
Flip angle $(\mathrm{FA})$ & $90^{\circ}$ \\
Fields of view $\left(\mathrm{FOV}_{\mathrm{x}}, \mathrm{FOV}_{\mathrm{y}}\right)$ & $240 \mathrm{~mm}, 240 \mathrm{~mm}$ \\
Resolution $(\Delta x, \Delta y)$ & $1.88 \mathrm{~mm}, 1.88 \mathrm{~mm}$ \\
Number of data points collected $\left(N_{\mathrm{x}}, N_{\mathrm{y}}\right)$ & 128,128 \\
Slice thickness $(\Delta z)$ & $5 \mathrm{~mm}$ \\
Number of slices & $30 \mathrm{with}$ the diffusion gradients in \\
Slice gap & 3 orthogonal directions \\
Number of acquisitions $\left(N_{\text {acq }}\right)$ & 0 \\
Swap read and phase encoding & 1 \\
Read direction & No (to decrease peripheral nerve \\
Saturation pulses & stimulation) \\
$b$-value & Left-right \\
$\delta$ & Not applicable \\
$\Delta$ & $1000 \mathrm{sec} / \mathrm{mm}^{2}$ \\
Scan time & $31 \mathrm{msec}$ \\
\hline & $36.6 \mathrm{msec}$ \\
\hline & $\sim 45 \mathrm{sec}$ \\
\hline
\end{tabular}

sequence first acquires a set of transverse $T_{2}$-weighted images (Beneviste et al., 1992) without the diffusion gradients (thus $b=0$ ). The diffusion gradients are then applied sequentially in three orthogonal axes in the directions of slice select, phase encoding, and read gradients to generate a set of 3 orthogonal-axis diffusion-weighted echo-planar images (EPI). The $b$-value (diffusion sensitivity) is $1000 \mathrm{sec} / \mathrm{mm}^{2}$. A set of isotropic images are calculated and displayed.

2. Obtain the DWI using parameters in Table A3.4.10 (sequence 11) to demonstrate that the lesion remains iso-intense to CSF.

3. Repeat steps 15 to 17 (sequence 5) in the Basic Protocol.

\section{EPIDERMOID/DERMOID}

Epidermoid and dermoid cysts are congenital lesions frequently found along the cerebellopontine angle, supra- and para-sellar regions, and middle cranial fossa. Epidermoid cysts appear similar, but not identical, in signal intensity to that of CSF and in the majority of cases epidermoids may be differentiated from arachnoid cysts by MRI. Dermoid cysts contain fat within them and fat suppressed images may be useful to confirm the contents of this lesion.

\section{Set up patient and equipment}

1. Repeat steps 1 to 10 and run sequences 1 to 5 (Basic Protocol).

2. Obtain fat suppressed $T_{1}$-weighted images (parameters in sequence 2 ) in the transverse or sagittal plane. 


\section{CHONDROSARCOMA/CHORDOMA}

Primary skull base lesions such as chordoma and chondrosarcoma can be infiltrative and spread to involve skull base foramina. The main purpose of imaging is to determine the full extent of the lesion (i.e., cranial nerve or meningeal involvement). The entire brain should always be imaged (sequences 1 to 5). Fat suppressed high-resolution imaging, including $T_{2}$-weighted images (sequence 6) and post-contrast $T_{1}$-weighted images (sequence 9) is essential when evaluating tumor involvement of skull base structures.

\section{Set up patient and equipment}

1. Use the same equipment and perform the same patient setup as for the previous method (see Basic Protocol, steps 1 to 10).

2. Run sequences 1 to 4 (Basic Protocol) to evaluate the entire brain.

3. For high-resolution imaging of the skull base, run sequence 6 according to Table A3.4.6 (transverse $T_{2}$-weighted images) and sequence 9 according to Table A3.4.9 (with imaging plane changed to transverse, and with read and phase encoding directions swapped). The entire extent of the lesion must always be included.

4. Repeat steps 15 and 16 in Basic Protocol.

5. Run post-contrast $T_{1}$-weighted scans in two planes (transverse and coronal) according to Table A3.4.9 adding the fat suppression option.

6. Run sequence 5 (post-contrast $T_{1}$-weighted scan of the entire brain, step 17) in the transverse plane.

\section{COMMENTARY}

\section{Background Information}

MRI is the preferred imaging modality for defining extra-axial tumors. Obtaining detailed information regarding the entire extent of disease is critical to the determination of appropriate treatment. High resolution computed tomography (CT) of the skull base is often an integral part of the work-up of a skull-base lesion. MRI provides superior contrast and is essential for documenting meningeal disease and spread along cranial nerves (see UNITS A7.1A7.4). Intravenous contrast is critical to help predict the full extent of disease.

\section{Critical Parameters and Troubleshooting}

Fluid attenuated inversion recovery (FLAIR) or post-contrast $T_{1}$-weighted images provide the greatest sensitivity in detecting extra-axial spread of tumor to the meninges. When evaluating the skull base, fat-suppressed high-resolution imaging (including $T_{2^{-}}$ weighted and post-contrast $T_{1}$-weighted images) is essential to clearly delineate the full extent of disease. With a resolution of less than $1 \mathrm{~mm}$ in the read and phase encoding directions, there is superb visualization of the fine intricate structures of the skull base (i.e., foramina and cranial nerves). Fat-suppressed $T_{1}$-weighted images are critical to evaluate for abnormal enhancement of cranial nerves, since high signal intensity on the $T_{1}$-weighted images from fat found along the skull base or chemical-shift artifact from fat-containing structures can obscure enhancement of cranial nerves. Inhomogeneities in the magnetic field can result in incomplete fat suppression or unintended suppression of water.

\section{Anticipated Results}

The goal of imaging when evaluating for extra-axial tumors is to accurately localize and detect the full extent of disease. Magnetic resonance imaging offers superb anatomical detail and tissue characterization of lesions. Fat suppressed high-resolution MR imaging is a key component of the skull-base examination. FLAIR images and post-contrast $T_{1}$-weighted images are most sensitive for detecting subarachnoid space disease.

\section{Time Considerations}

The majority of these protocols should take $\sim 30$ min to complete. 


\section{Literature Cited}

Beneviste, H., Hedlund, L., and Johnson, G. 1992. Mechanism of detection of acute cerebral ischemia in rats by diffusion-weighted magnetic resonance microscopy. Stroke 23:746-754.

Shellock, F.G. 1996. Pocket Guide to MR Procedures and Metallic Objects. (F.G. Shellock, ed.). Lippincott-Raven, Philadelphia.

Singer, M.B., Atlas, S.W., and Drayer, B.P. 1998. Subarachnoid space disease: Diagnosis with fluid-attenuated inversion-recovery MR imaging and comparison with gadolinium-enhanced spin echo MR imaging-blinded reader study. Radiology 208:417-422.

Contributed by Annette O. Nusbaum

New York Presbyterian Hospital

New York, NY

Scott W. Atlas

Stanford University Medical Center Stanford, CA
Cerebral Neoplastic Disease

A3.4.11 